\title{
Design and Performance of the Antenna-Coupled Lumped-Element Kinetic Inductance Detector
}

\author{
P. S. Barry ${ }^{1,2}$ - S. Doyle ${ }^{2}$ - A. L. Hornsby ${ }^{2}$. \\ A. Kofman ${ }^{3}$ - E. Mayer ${ }^{1}$ - A. Nadolski ${ }^{3}$. \\ Q. Y. Tang ${ }^{1}$ - J. Vieira ${ }^{3}$ - E. Shirokoff ${ }^{1}$
}

Received: 3 November 2017 / Accepted: 30 April 2018 / Published online: 11 May 2018

(C) The Author(s) 2018

\begin{abstract}
Focal plane arrays consisting of low-noise, polarisation-sensitive detectors have made possible the pioneering advances in the study of the cosmic microwave background $(\mathrm{CMB})$. To make further progress, the next generation of CMB experiments (e.g. CMB-S4) will require a substantial increase in the number of detectors compared to current instruments. Arrays of kinetic inductance detectors (KIDs) provide a possible path to realising such large-format arrays owing to their intrinsic multiplexing advantage and relative cryogenic simplicity. In this paper, we report on the design of a variant of the traditional KID design: the antenna-coupled lumpedelement KID. A polarisation-sensitive twin-slot antenna placed behind an optimised hemispherical lens couples power onto a thin-film superconducting microstrip line. The power is then guided into the inductive section of an aluminium KID, where it is absorbed and modifies both the resonant frequency and quality factor of the KID. We present the various aspects of the design and preliminary results from the first set of seven-element prototype arrays and compare to the expected modelled performance.
\end{abstract}

Keywords $\mathrm{CMB} \cdot$ Instrumentation $\cdot$ Kinetic inductance detectors

P. S. Barry

barryp@uchicago.edu

1 Kavli Institute for Cosmological Physics, University of Chicago, Chicago, IL, USA

2 School of Physics and Astronomy, Cardiff University, The Parade, Cardiff CF24 3AA, UK

3 Astronomy Department, University of Illinois, 1002 W. Green St., Urbana, IL 61801, USA 


\section{Introduction}

Kinetic inductance detectors (KIDs) [1] are now considered a compelling alternative technology for large-format focal plane arrays. A number of projects operating at millimetre (NIKA-2 [2], TolTEC [5], SuperSpec [3,4]) and submillimetre (SpaceKIDs [6], BLAST-TNG [7]) wavelengths will help to deliver the first large-scale on-sky demonstration of KID arrays, whose promise of high multiplexing factors and simple fabrication provides a path to reduced cost and complexity of future large-format focal plane instruments.

However, to date, experiments designed to measure the cosmic microwave background ( $\mathrm{CMB}$ ) have been led by instruments based on arrays of transition edge sensors (TESs). State-of-the-art CMB experiments are at 'stage 3', with focal planes containing 5000-15,000 TESs [13-15] operating close to the background-limit. In this case, further improvements in sensitivity can only be achieved by increasing total focal plane area and number of detectors. Furthermore, motivated by the desire to constrain foreground contamination, current experiments have implemented integrated filtering schemes, where each pixel in the focal plane accepts a wide-band signal that is divided into multiple sub-bands with lithographic on-chip filters and sensed with separate detectors. The required increase in the number of detectors and corresponding readout needed to achieve the goals of the future experiments is a significant technological challenge. Estimates indicate that CMB-S4 will require a minimum of 500,000 background-limited detectors across $\sim 10$ ground-based telescopes [17]. It is here where the advantages of KIDs could play an important role in realising the next generation of $\mathrm{CMB}$ experiments.

A KID is a superconducting, high-Q resonator that senses the change in complex surface impedance of the superconducting film upon photon absorption. Generally, there are two implementations of thin-film superconducting resonators used as the KID transducer, distributed resonators (mKIDs) [1] that are based on half-wave, or quarterwave planar transmission line resonators, and lumped-element resonators (leKIDs) that combine discrete and separate inductive and capacitive elements to form a standard LC circuit [16]. The ability to separate the inductive and capacitive elements provides a degree of versatility that can be used to optimise the device geometry for a specific application. Furthermore, the constant current density across the inductor gives a spatially uniform responsivity, and the design of the inductor can be arranged to form an efficient free-space absorber. This additional degree of freedom is a major advantage of the leKID and is compatible with various radiation coupling schemes [7,8].

Despite these advantages, the traditional leKID design is not immediately compatible with the chip-level filtering schemes envisioned for future CMB experiments. In this paper, we report on the design of the antenna-coupled leKID, which provides a method to combine the advantages of the traditional leKID design with on-chip band definition to construct highly-multiplexed, polarisation-sensitive, multi-chroic focal planes. 

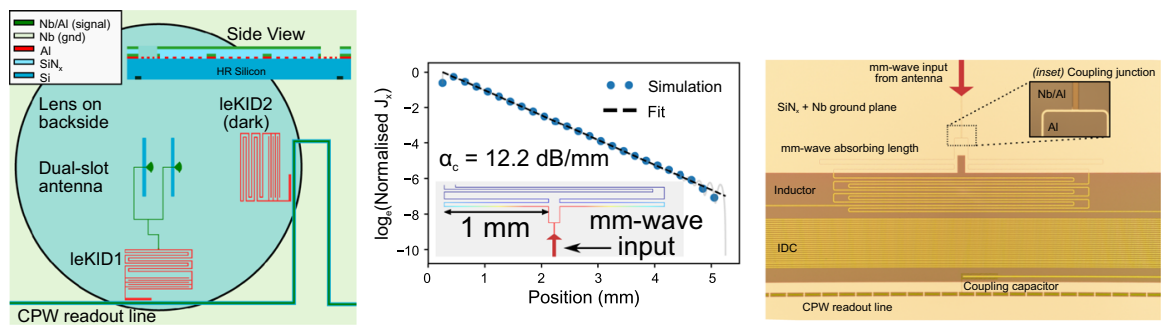

Fig. 1 (Left) Schematic of a single pixel from the prototype array, (middle) Simulated current density along the lossy $\mathrm{Al}$ microstrip line at $150 \mathrm{GHz}$, (right) optical microscope image of the first fabricated arrays (Color figure online)

\section{Millimetre-Wave Coupling}

The design of the antenna-coupled leKID is based around the coupling mechanism from the mm-wave feedline into the detector. Radiation is coupled onto a thin-film superconducting microstrip line using a lens-coupled twin-slot antenna. We use an inverted microstrip design [3], where the circuit layer is deposited onto a clean, highresistivity silicon surface, and is covered by the dielectric and groundplane layers, shown in Fig. 1. The principal advantage in adopting this design is that deposition of the detector Al layer is the first process in the fabrication procedure. This allows maximal control over the substrate preparation which has been shown to be critical to minimising the contributions to the loss and noise originating from two-level fluctuations. Full details on the fabrication procedure are given in Tang et al. [9].

The microstrip line is made from an $\mathrm{Al} / \mathrm{Nb}$ bilayer of thickness $50 / 250 \mathrm{~nm}$, with $T_{c}=6.8 \mathrm{~K}\left(\nu_{\text {gap }} \approx 500 \mathrm{GHz}\right)$. The bilayer is used to simplify the fabrication of the mm-wave-to-KID junction. A 500-nm PECVD $\mathrm{SiN}_{\mathrm{x}}$ layer forms the dielectric insulator and a final $250-\mathrm{nm} \mathrm{Nb}$ layer acts as the groundplane, into which the antenna slots are patterned. The microstrip linewidth should remain small in order to minimise radiation losses and is largely determined by lithographic tolerances. For our design, we use a 4- $\mu \mathrm{m}$ microstrip line that results in characteristic impedance of $Z_{0}=17.6 \Omega$. The microstrip from the two slots is then combined into a single line that feeds the leKID. In the interest of understanding the fundamental device performance, we have chosen to not include any band-defining components in the prototype devices. However, it is trivial to add any planar filter into the design at this point and is something we are working to include subsequent device iterations.

At the leKID, the $\mathrm{Al} / \mathrm{Nb}$ microstrip line is split and continues as an Al-only microstrip line (cf. inset of Fig. 1, right) that doubles as the leKID inductor. The detector is patterned into the base $\mathrm{Al}$ layer and provides a simple way to achieve a low-loss galvanic contact between the input transmission line and leKID inductor. From a mm-wave perspective, the leKID inductor acts as a low-loss transmission line with an impedance chosen to ensure the input impedance looking into the inductor is matched (i.e. $Z_{0 \text {,out }}=2 Z_{0 \text {,in }}$ ). As this junction is non-resonant, it is inherently wideband. However, because the $\mathrm{Al}$ microstrip has nonzero loss at frequencies above the gap frequency $\left(v_{\text {gap }} \approx 100 \mathrm{GHz}\right.$ ), the characteristic impedance has a nonzero imagi- 
nary component, which presents a small impedance mismatch at the junction. For the 4- $\mu \mathrm{m}$ Al microstrip, we calculate $Z_{0, \mathrm{Al}}=17.6+j 3 \Omega$.

Planar EM simulations suggest that the match of this T-junction can be better than 20 $\mathrm{dB}$ cross the entire millimetre-wave band $(90-300 \mathrm{GHz})$. The simulation defines each metal layer by a sheet impedance, which for $\mathrm{Al}$ is well characterised at frequencies both below and above the gap frequency. For modelling the Al/Nb bilayer, we assume that the sheet impedance is similar to bare $\mathrm{Nb}$. While we expect this assumption to be reasonable, we are in the process of measuring this quantity to inform future design iterations. Furthermore, we expect that the $\mathrm{Nb}$ layer will proximitise the $\mathrm{Al}$ in the vicinity of the junction and provide a smoother impedance transition relative to our current simulations.

Once the mm-wave power is travelling in the inductor, the amplitude is expected to decrease exponentially along the inductor length. To ensure that all of the power is absorbed in the inductor, the length of the absorbing section must be chosen appropriately. The attenuation length $\left(\alpha_{c}\right)$ for the $\mathrm{Al}$ microstrip line can be estimated using the standard expression for microstrip conductor loss in $\mathrm{dB} / \mathrm{mm}$,

$$
\alpha_{c}=8.686 \cdot \frac{R_{s}}{2 Z_{0} w} .
$$

For a measured value of $R_{s}^{\mathrm{Al}}=0.2 \Omega / \mathrm{sq}$ for a $50 \mathrm{~nm}$ film, $Z_{0}=17.6 \Omega$, and linewidth $w=4 \mu \mathrm{m}$, we estimate $\alpha_{c} \approx 12.3 \mathrm{~dB} / \mathrm{mm}$. Comparison with a $2 \mathrm{D}$ simulation of the current density profile gives value of $12.2 \mathrm{~dB} / \mathrm{mm}$ (cf. Fig. 1), in good agreement with the analytic estimate. This value of $\alpha_{c}$ suggests that a length of greater than a couple of millimetres will be sufficient to absorb the incoming signal. For the prototype design, we allow $3 \mathrm{~mm}$ length in each arm before the microstrip line is terminated by removing the groundplane and $\mathrm{SiN}_{\mathrm{x}}$. From the mm-wave perspective, the abrupt impedance mismatch will reflect any power that reaches the termination and provide a second pass for absorption.

In the first design iteration, the main goal was to demonstrate the microstrip-KID coupling architecture, and the design of the inductor was not explicitly optimised to minimise direct absorption of radiation. To address this in future devices, there are two paths we plan to take to mitigate this problem: 1) we will form a hybrid-inductor, in which the exposed region of the inductor is made from $\mathrm{Nb} / \mathrm{Al}$, and the portion underneath the groundplane remains $\mathrm{Al}$ and 2) perform a direct optimisation of the inductor design through EM simulation. The ability to optimise each component of the detector independently demonstrates an important advantage of the antenna-coupled leKID design.

\section{RF Design}

The design of the leKID is subject to a number of constraints unique to this architecture. As discussed above, the portion of the inductor where the mm-wave absorption takes place remains under the groundplane and acts as a lossy microstrip line, absorbing and attenuating the mm-wave signal along the length. The rest of the inductor, and 
inter-digital capacitor (IDC), is uncovered, as shown in the right panel of Fig. 1. The motivation behind removing the groundplane and dielectric above the length of inductor not acting as an absorber is to reduce the distributed capacitance to ground, which would otherwise dominate the overall capacitance of the resonator and modify the current distribution across the inductor. Furthermore, the dielectric above the IDC is removed to reduce the effect of TLS loss and fluctuation noise.

The mm-wave input microstrip is connected to the centre of the inductor at the voltage node of the resonator. Placing the microstrip at this location is not expected to affect the RF properties of the resonator as there is negligibly small voltage to drive any appreciable current out of the resonator. Moreover, the direct capacitive connection to ground can potentially enhance the uniformity of resonator frequencies by reducing the effect of variable stray capacitances to external circuit components (e.g. sample box).

We calculate the expected sensitivity using a modified responsivity model based on the total number of quasiparticles. We assume a optical loading typical of ground-based CMB experiments of between 1 and $10 \mathrm{pW}$ [18], which sets the background-limited $\mathrm{NEP}_{\gamma}^{2}=2 h v P_{\text {abs }}(1+\bar{n})$. From the expected power loading, we calculate the total number of quasiparticles that includes, self-consistently, contributions from both the optical signal and thermal excitations present at a base temperature of $250 \mathrm{mK}$. We then calculate an effective quasiparticle temperature that is then used in place of the base temperature. The left panel of Fig. 2 shows the various contributions to the noise for a nominal inductor volume of $4800 \mu \mathrm{m}^{3}$ and indicates that the device should remain background-limited for the expected optical loading.

As only a portion of the inductor is used for mm-wave absorption, the amount of additional inductance will act to reduce the responsivity. The normal method to account for this reduction would be incorporate a partial kinetic inductance fraction $\left(\alpha_{k}^{*}\right)$. However, in this case, the calculation of $\alpha_{k}^{*}$ is not straightforward, as quasiparticles generated in the mm-wave absorbing section are able to diffuse to the remainder of the inductor, and still contribute to the responsivity. The diffusion length is expected to be on the order of a few $\mathrm{mm}[19,20]$ for typically measured quasiparticle lifetimes in Al films. Therefore, we have included a range of inductor volumes around the nominal value that will allow us to quantify the magnitude of this effect through a measure of the the optical responsivity. We target resonant frequencies in the range $500-1000 \mathrm{MHz}$, while other resonator parameters remain identical for each device, with IDC linewidth/gap $=4 / 10 \mu \mathrm{m}$ and a coupling $Q_{c}=10^{5}$.

\section{Preliminary Results}

In this section, we present a preliminary characterisation of the microwave performance of the detectors tested in a dark environment. We place the devices into a gold-plated OFHC copper sample box, mounted in situ with the lens array using our novel alignment scheme, as described in Tang et al. [9]. The box is mounted onto the mixing chamber of a dilution refrigerator with a base temperature of $10 \mathrm{mK}$. The resonant frequency and quality factor are extracted from fits to VNA sweeps, and a standard single-tone homodyne configuration is used to characterise the noise performance [1]. 

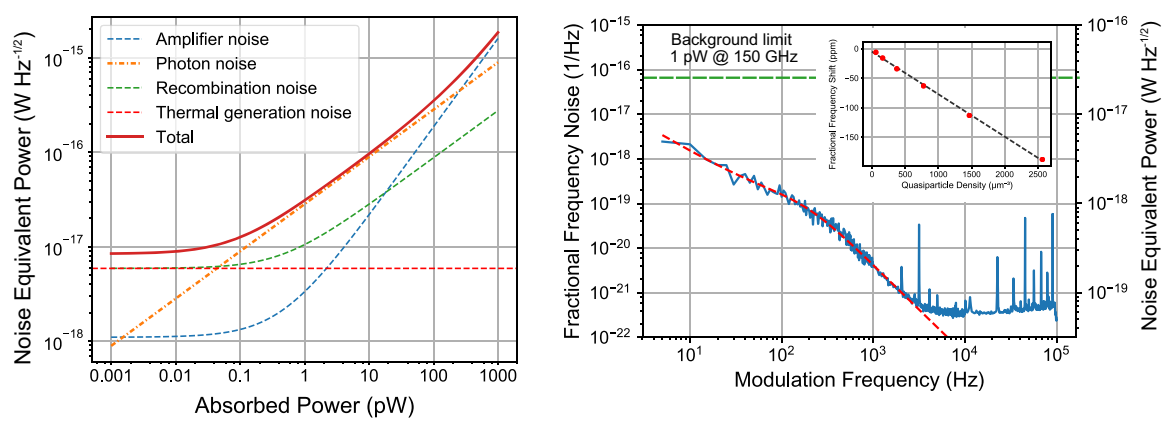

Fig. 2 (Left) Calculated expected contributions to the noise equivalent power (NEP) as a function of optical load at $T_{b}=230 \mathrm{mK}$, (right) measured noise spectrum at $100 \mathrm{mK}$ with a fit (red dashed line) to extract the resonator time constant. Right axis is an estimate of the electrical NEP using a measure of the dark responsivity (inset). Note that the data have not been corrected for time-constant roll-off. Dashed green line is the expected background-limited NEP for $1 \mathrm{pW}$ optical load at $150 \mathrm{GHz}$ (Color figure online)

We measure internal quality factors $\left(Q_{i}\right)$ in excess of $0.5 \times 10^{6}$ for all resonators without the $\mathrm{SiN}_{\mathrm{x}}$ layer over the capacitors [10]. This is an important result for this design as it demonstrates that placing $\mathrm{SiN}_{\mathrm{x}}$ onto the inductive section of a leKID does not have a significant impact on $Q_{i}$. In terms of scaling to large arrays, $Q_{i}>2 \times 10^{5}$ is well within the requirements for multiplexing ratios of above $10^{3}$ using existing readout hardware [21].

To estimate the performance of the prototype device from a detector perspective, we measure the electrical noise equivalent power (NEP). It has recently been shown that an estimate of electrical NEP provides an accurate indication of the optical performance for devices where the contribution to the responsivity is constant across the absorbing volume [12]. For a leKID, this is automatically satisfied as the current density across the inductor is constant.

The electrical NEP is calculated from Eq.2. An estimate of the responsivity is determined from a fit to the resonator response as a function base temperature. The noise level $\left(S_{x x}\right)$ and quasiparticle lifetime $\left(\tau_{q p}\right)$ are evaluated from the noise spectrum, and along with known material and geometric parameters, the NEP is calculated from [11]

$$
\mathrm{NEP}_{\delta x}=\sqrt{S_{x x}} \cdot\left[\frac{d x}{d n_{q p}} \cdot \frac{\eta_{\mathrm{pb}} \tau_{q p}}{\Delta_{0} V_{L}}\right]^{-1}
$$

where $x=\delta f / f_{r}$ is the fractional frequency shift, $\eta_{\mathrm{pb}}=0.57$ is the pair breaking efficiency, $\Delta_{0}$ is the low temperature superconducting gap, and the equilibrium quasiparticle density is given by

$$
n_{q p}=2 N_{0} \sqrt{2 \pi k_{B} T_{c} \Delta_{0}} \exp \left(-h v / k_{B} T\right)
$$

The inset of the right panel of Fig. 2 shows the measured $\delta x$ vs $n_{q p}$ for a representative resonator. A linear fit to the response is used to extract the quasiparticle responsivity. The fit range is chosen to capture only the higher temperature behaviour where 
the quasiparticle response dominates over the dielectric response. The quasiparticle lifetime is extracted from a fit to roll-off in the noise spectrum. The measured $\tau_{q p}=$ $850 \mu \mathrm{s}$ at $100 \mathrm{mK}$, which is significantly longer than the expected resonator ring-down time $\tau_{\text {res }}=2 Q_{r} / \omega_{0} \approx 50 \mu \mathrm{s}$.

The right panel of Fig. 2 shows the measured noise spectrum for a resonator at $100 \mathrm{mK}$. The $1 / f^{n}$ spectral shape indicates that the noise performance at low modulation frequencies is most likely limited by TLS fluctuation noise. In subsequent devices, we will prioritise further reducing this level by modifying the capacitor geometry and improving the substrate preparation before film deposition.

The NEP derived from the responsivity is shown on the right-hand $y$-axis of Fig. 2. The dashed line is the expected background-limited NEP for the expected detector loading of $1 \mathrm{pW}$ and indicates that for the expected loading from a ground-based CMB experiment, the performance of these devices will be firmly background-limited, with a device-noise $1 / f^{n}$ knee below $1 \mathrm{~Hz}$.

\section{Conclusions and Future Work}

We have introduced the concept and design of the antenna-coupled leKID, which will enable the advantages of the leKIDs to be combined with existing multi-chroic on-chip mm-wave filtering circuits that will be crucial for future CMB experiments. The measured electrical NEP is promising, with a significant amount of clearance to the expected level for background-limited sensitivity. Based on these data, this detector architecture appears to be an interesting candidate for ground-based CMB experiments with detector power loading of $1-10 \mathrm{pW}$. The addition of optical data and characterisation will determine the ultimate performance of this device, and we plan to report on this soon.

Acknowledgements This material is based in part upon work supported by the National Science Foundation under Grant Number 1554565. This work also made use of the Pritzker Nanofabrication Facility of the Institute for Molecular Engineering at the University of Chicago, which receives support from SHyNE, a node of the National Science Foundations National Nanotechnology Coordinated Infrastructure (NSF NNCI-1542205). We acknowledge support from the Science and Technology Facilities Council (STFC) Consolidated Grant Ref: ST/N000706/1 for supporting this work in the UK.

Open Access This article is distributed under the terms of the Creative Commons Attribution 4.0 International License (http://creativecommons.org/licenses/by/4.0/), which permits unrestricted use, distribution, and reproduction in any medium, provided you give appropriate credit to the original author(s) and the source, provide a link to the Creative Commons license, and indicate if changes were made.

\section{References}

1. P.K. Day, H.G. Leduc, B.A. Mazin, A. Vayonakis, J. Zmuidzinas, A broadband superconducting detector suitable for use in large arrays. Nature 425(6), 817-821 (2003)

2. M. Calvo et al., The NIKA2 instrument, a dual-band kilopixel KID array for millimetric astronomy. J. Low Temp. Phys. 184(3), 816-823 (2016)

3. E. Shirokoff et al., Design and performance of superspec: an on-chip, KID-based, mm-wavelength spectrometer. J. Low Temp. Phys. 176(5), 657-662 (2014)

4. J. Wheeler et al., SuperSpec: development towards a full-scale filter bank. Proc. SPIE 9914, K9 (2016) 
5. http://toltec.astro.umass.edu/about.php

6. M. Griffin et al., SPACEKIDS: kinetic inductance detectors for space applications. Proc. SPIE 9914, 11 (2016)

7. N. Galitzki et al., The next generation BLAST experiment. J. Astron. Instrum. 3(2), 1440001 (2014)

8. B.A. Mazin et al., ARCONS: a 2024 pixel optical through near-IR cryogenic imaging spectrophotometer. Publ. Astron. Soc. Pac. 125(933), 1348-1361 (2013)

9. Q.Y. Tang et al., Fabrication of antenna-coupled KID array for Cosmic Microwave Background detection. J. Low Temp. Phys. (2018) (this Special Issue LTD17)

10. A. Hornsby et al., An initial investigation into the susceptibility of antenna-coupled LEKIDs to two level system effects. J. Low Temp. Phys. (2018) (this Special Issue LTD17)

11. J. Baselmans et al., Noise and sensitivity of aluminum kinetic inductance detectors for sub-mm astronomy. J. Low Temp. Phys. 151(1), 524-529 (2008)

12. R.M.J. Janssen et al., Equivalence of optical and electrical noise equivalent power of hybrid NbTiN-Al microwave kinetic inductance detectors. Appl. Phys. Lett. 105(19), 193504 (2014)

13. Z. Ahmed et al., BICEP3: a $95 \mathrm{GHz}$ refracting telescope for degree-scale CMB polarization, in Millimeter, Submillimeter, and Far-Infrared Detectors and Instrumentation for Astronomy VII. SPIE Proceedings (International Society for Optics and Photonics, 2014), p. 91531N

14. B.A. Benson et al., SPT-3G: a next-generation cosmic microwave background polarization experiment on the South Pole telescope. SPIE Astron. Telesc. Instrum. 9153, 91531P91531P21 (2014)

15. R.J. Thornton et al., The Atacama Cosmology Telescope: the polarization-sensitive ACTpol instrument. Astrophys. J. Suppl. Ser. 227(2), 21 (2016)

16. S.M. Doyle et al., Lumped element kinetic inductance detectors. J. Low Temp. Phys. 151(1-2), 530-536 (2008)

17. K.N. Abazajian et al., CMB-S4 Science Book, 1st edn. (2016). arXiv: 1610.02743

18. BICEP Collaboration et al., Antenna-coupled TES bolometers used in BICEP2, keck array, and spider. Astrophys. J. 812(2), 176 (2015)

19. S.Y. Hsieh, J.L. Levine, Diffusion of quasiparticles in superconducting aluminum films. Phys. Rev. Lett. 20(26), 1502-1504 (1968)

20. M. Loidl et al., Quasiparticle diffusion over several $\mathrm{mm}$ in cryogenic detectors. Nucl. Instrum. Methods Phys. Res. Sect. A 465(2-3), 440-446 (2001)

21. S. Gordon et al., An open source, FPGA-based LeKID readout for BLAST-TNG: pre-flight results. J. Astron. Instrum. 05(04), 1641003 (2016) 